\title{
A smoothing multidimensional filter method for nonlinear complementarity problems
}

\author{
Wu Congwei \\ Xi'an Research Institute of High-tech \\ Tongxin Road No.2, 2102 Team, \\ Baqiao District, \\ Xi'an City, Shaanxi Province, \\ P.R.China \\ Email:wucongweihome@sina.com
}

\author{
Cao Jiping \\ Xi'an Research Institute of High-tech \\ Tongxin Road No.2, Baqiao District, \\ Xi'an City, Shaanxi Province, \\ P.R.China \\ Email: xacjp123@163.com
}

\author{
Zhu Yahong \\ Xi'an Research Institute of High-tech \\ Tongxin Road No.2, 2102 Team, \\ Baqiao District, \\ Xi'an City, Shaanxi Province, \\ P.R.China \\ Email: zyhbingchen@163.com
}

\begin{abstract}
A smoothing multidimensional filter method for solving NCP is proposed, and the solution of the complementarity problems on the framework of the filter-trust-region method is obtained. The new algorithm does not depend on any extra restoration procedure and the results of numerical experiments show its efficiency.
\end{abstract}

Keywords-Multidimensional filter techniques; Nonlinear complementarity problems; filter-trust-region method

\section{INTRODUCTION}

Let $F(x): \mathfrak{R}^{n} \rightarrow \mathfrak{R}^{n}$ be a continuous differentiable function. The nonlinear complementarity problem (NCP) is to find a vector $x \in \mathfrak{R}^{n}$ such that

$$
x \geq 0, \quad F(x) \geq 0, \quad x^{T} F(x)=0,
$$

For convenience, denote $I=\{1,2, \ldots, n\}$. Throughout this paper, $\|\cdot\|$ denotes the Euclidean norm.

The traditional approach for NCP involves reformulating the problem as a constrained or unconstrained optimization problem. We discuss for solving this optimization based on the class of trust-region methods and also on that of multidimensional filter methods introduced by Gould and Sainvitu [1].

Definition 1 A function $\phi: \mathfrak{R}^{2} \rightarrow \mathfrak{R}$ is said to be an NCP function if it possesses the following characterization $\phi(a, b)=0 \Leftrightarrow a \geq 0, b \geq 0$ and $a b=0$.

In this paper, we will use Fischer-Burmeister functions $\phi: \Re^{2} \rightarrow \Re$ defined by $\phi(a, b):=\sqrt{a^{2}+b^{2}}-(a+b)$

It is obviously that $\phi$ is not differentiable at zero point, which is difficult to get the global convergence results, so we use the following smooth approximation for the FischerBurmeister function,

$$
\phi_{\mu}(a, b):=\sqrt{a^{2}+b^{2}+\mu^{2}}-(a+b)
$$

where $\mu \geq 0$ is a smooth parameter. Then NCP can be approximated by the following nonlinear least square problems,

$$
\min _{x \geq 0} f_{\mu}(x):=\frac{1}{2} \Phi_{\mu}(x)^{T} \Phi_{\mu}(x)
$$

where

$$
\Phi_{\mu}(x)=\left(\begin{array}{c}
\phi_{\mu}\left(x_{1}, F_{1}(x)\right) \\
\vdots \\
\phi_{\mu}\left(x_{n}, F_{n}(x)\right)
\end{array}\right)
$$

Obviously, if the complementarity problem (1) is solvable, then the minimization problem (2) and (1) are equivalent when the parameter $\mu$ tends to zero. Throughout this paper, to simplify notation we will use the abbreviations $g_{\mu}(x)=\nabla_{x} f_{\mu}(x)$. Beside, $g_{\mu, i}(x)$ denote the i-th component of $g_{\mu}(x)$.

\section{The Multidimensional FiLter Method}

The projected gradient of the objective function $\mathrm{f}$ ( $\mathrm{x}$ ) into the feasible set of the problem (2) is defined componentwise by

$$
\bar{g}_{i}(x)= \begin{cases}g_{i}(x), & x_{i} \geq g_{i}(x), \\ x_{i}, & x_{i}<g_{i}(x),\end{cases}
$$

where $i \in I$.

Note that the problem (2) is a nonlinear optimization problem with a positive parameter $\mu$, so we can use $x_{\mu}^{*}$ denote the KKT point of the problem (2), and then we have the following Conclusion.

Lemma 1 For all $\mu>0$, a point $x_{\mu}^{*}$ is a KKT point for the problem (2) if and only if $\bar{g}_{\mu}\left(x_{\mu}^{*}\right)=0$.

Lemma 2 Assume that $F(x)$ is a twice-continuously differentiable function on an closed and bounded set, then the $K K T$ point of (2) converges to the KKT point of the following problem

$$
\min _{x \geq 0} f_{0}(x):=\frac{1}{2} \Phi_{0}(x)^{T} \Phi_{0}(x)
$$

when the parameter $\mu$ tends to zero.

To solve the optimization problem (2), we compute a trial step $d_{k}$ at a given iterate $x_{k}$ by the following trust-region 
subproblem

$$
\begin{array}{ll}
\min & Q_{k}(d)=f_{\mu_{k}}\left(x_{k}\right)+\nabla_{x} f_{\mu_{k}}\left(x_{k}\right)^{T} d+\frac{1}{2} d^{T} B_{k} d, \\
\text { s.t. } \quad & x_{k}+d \geq 0, \\
& \|d\|_{\infty} \leq \Delta_{k}
\end{array}
$$

Where $\Delta_{k}$ is the trust region radius, $B_{k}=\nabla_{x} \Phi_{\mu_{k}}\left(x_{k}\right) \nabla_{x} \Phi_{\mu_{k}}\left(x_{k}\right)^{T}$, and

$$
\nabla_{x} \Phi_{\mu}(x)=\left(\begin{array}{ccc}
\frac{\partial \Phi_{\mu, 1}(x)}{\partial x_{1}} & \ldots & \frac{\partial \Phi_{\mu, n}(x)}{\partial x_{1}} \\
\vdots & \ddots & \vdots \\
\frac{\partial \Phi_{\mu, 1}(x)}{\partial x_{n}} & \ldots & \frac{\partial \Phi_{\mu, n}(x)}{\partial x_{n}}
\end{array}\right)
$$

The positive parameters $\mu_{k}$ tends to zero during the iterate of algorithm. A trial point $x_{k}^{+}$is then computed by the trial step $d_{k}$, denote $x_{k}^{+}=x_{k}+d_{k}$.

In our context, Our aim is to encourage convergence to first-order critical points by driving every component of the projected gradient $\bar{g}_{\mu}(x)=\left(\bar{g}_{\mu, 1}(x), \bar{g}_{\mu, 2}(x), . \bar{g}_{\mu, h}(x)\right)^{T}$ to zero.

Definition 2 A iterate point $x_{k}$ is said to dominate another point $x_{l}$ if and only if $\left|\bar{g}_{\mu_{k-1}, i}\left(x_{k}\right)\right| \leq\left|\bar{g}_{\mu_{1-1}, i}\left(x_{l}\right)\right|, \forall i \in I$.

Definition 3 A filter set. $\mathscr{T}$ is a set of points such that no pair dominates any other.

We say that a new trial point $x_{k}^{+}$is acceptable for the filter $\mathscr{T}_{k}$ if and only if

$$
\forall x_{l} \in \mathscr{F}_{k}, \exists j \in I,\left|\bar{g}_{\mu_{k}, j}\left(x_{k}^{+}\right)\right| \leq\left|\bar{g}_{\mu_{l-1}, j}\left(x_{l}\right)\right|-\gamma_{g}\left\|\bar{g}_{\mu_{l-1}}\left(x_{l}\right)\right\| \text {, }
$$

Where $\gamma_{g}=(0,1 / \sqrt{n})$.

If an iterate $x_{k}$ is acceptable for the filter $\widetilde{T}_{k}$, we add it to the filter and remove from it every $x_{l} \in \mathscr{F}_{k}$, such that $\left|\bar{g}_{\mu_{k}, i}\left(x_{k}^{+}\right)\right| \leq\left|\bar{g}_{\mu_{l-1}, i}\left(x_{l}\right)\right|$ for all $i \in I$, i.e.

$$
\mathscr{F}_{k+1}=\mathscr{T}_{k} \cup\left\{x_{k}^{+} \backslash \mathscr{V}_{k}\right\}
$$

where,

$$
\mathscr{V}_{k}=\left\{x_{l} \in \mathscr{T}_{k}|| \bar{g}_{\mu_{k}, i}\left(x_{k}^{+}\right)|\leq| \bar{g}_{\mu_{l-1}, i}\left(x_{l}\right) \mid, \forall i \in I\right\}
$$

Note that the element of the set $\widetilde{T}_{k}$ is not $x_{k}^{+}$ but $\left(\left|\bar{g}_{\mu_{k}, 1}\left(x_{k}^{+}\right)\right|, \ldots,\left|\bar{g}_{\mu_{k}, n}\left(x_{k}^{+}\right)\right|\right)^{T}$. when we say a new trial point $x_{k}^{+}$is acceptable for the filter $\mathscr{T}_{k}$. Nevertheless, it is convenient to say that we add point $x_{k}^{+}$into $\mathscr{T}_{k}$.

\section{The Multidimensional Filter Algorithm}

At each step, after a subproblem is solved, the filter and traditional trust region criterion are all employed to determine whether to accept the trial point or not. In trust region criterion, if there is a good agreement between the model and the objective function value at the current trail point $x_{k}^{+}=x_{k}+d_{k}$, it is said to be successful iteration; otherwise, it is said to be unsuccessful iteration.

In following algorithm, the multidimensional filter criterion is a relaxation for the trust region criterion to a certain extent because we even accept the set of iterations which is not accepted by trust-region criterion but filter criterion. NCP

Algorithm 1 The Multidimensional Filter Algorithm for

Step 0: Initialization. An initial point and an initial trustregion radius $\Delta_{0}$ are Let an initial point $x_{0}$, an initial trustregion radius $\Delta_{0}>0$, and an initial filter set $\mathscr{F}_{0}=\left(10^{5}, \ldots, 10^{5}\right)^{T}$ be given, as well as constants $\gamma_{g}=(0,1 / \sqrt{n}), 0<\gamma_{1}<\gamma_{2}<1<\gamma_{3}, 0<\Delta_{0}<\Delta_{\max }$. Compute $f_{\mu_{0}}\left(x_{0}\right), g_{\mu_{0}}\left(x_{0}\right), \bar{g}_{\mu_{0}}\left(x_{0}\right), B_{0}$, set $k_{0}:=0$.

Step 1: Test for optimality. If $\left\|\bar{g}_{\mu_{k}}\left(x_{k}\right)\right\|+\mu_{k}<\varepsilon$, stop.

Step 2: Determine a trial step. Compute a solution $d_{k}$ of the subproblem (6).

Step 3: If $d_{k}=0$, set $x_{k+1}=x_{k}, \mu_{k+1}=\theta \mu_{k}, B_{k+1}=B_{k}$, set $k:=k+1$, and go to Step1; else set $x_{k}^{+}=x_{k}+d_{k}$, and compute $f_{\mu_{k}}\left(x_{k}^{+}\right), g_{\mu_{k}}\left(x_{k}^{+}\right)$.

Step 4: Test for optimality. If $\left\|\bar{g}_{\mu_{k}}\left(x_{k}^{+}\right)\right\|+\mu_{k}<\varepsilon$, stop; else, compute

$$
\rho_{k}=\frac{f_{\mu_{k}}\left(x_{k}\right)-f_{\mu_{k}}\left(x_{k}^{+}\right)}{Q_{k}(0)-Q_{k}\left(d_{k}\right)}
$$

Step 5: Tests to a accept the trial step.

- If $\rho_{k} \geq \eta_{1}$, set $x_{k+1}=x_{k}^{+}, \widetilde{T}_{k+1}=\mathscr{T}_{k} \cup\left\{x_{k}^{+} \backslash \mathscr{O}_{k}\right\}$;

- Else if $\rho_{k}<\eta_{1}$ and $x_{k}^{+}$is acceptable for the filter $\mathscr{T}_{k}$, set $x_{k+1}=x_{k}^{+}, \mathscr{T}_{k+1}=\mathscr{T}_{k} \cup\left\{x_{k}^{+} \backslash \mathscr{V}_{k}\right\}$,

- Otherwise, set $x_{k+1}=x_{k}, \mathscr{T}_{k+1}=\mathscr{T}_{k}$.

Step 6: Update the trust-region radius and the smooth parameter.

$$
\begin{aligned}
& \Delta_{k+1}= \begin{cases}{\left[\gamma_{1} \Delta_{k}, \gamma_{2} \Delta_{k}\right],} & \text { if } \rho_{k}<\eta_{1}, \\
\left(\gamma_{2} \Delta_{k}, \Delta_{k}\right], & \text { if } \rho_{k} \in\left[\eta_{1}, \eta_{2}\right), \\
\min \left\{\Delta_{\max }, \gamma_{3} \Delta_{k}\right\}, & \text { if } \rho_{k} \geq \eta_{2} .\end{cases} \\
& \mu_{k+1}= \begin{cases}\theta \mu_{k}, & \text { if } \mu_{k}>0.1\left\|\bar{g}_{\mu_{k}}\left(x_{k+1}\right)\right\|, \\
\mu_{k}, & \text { otherwise. }\end{cases}
\end{aligned}
$$

Step 7: Compute $f_{\mu_{k+1}}\left(x_{k+1}\right), g_{\mu_{k+1}}\left(x_{k+1}\right), \bar{g}_{\mu_{k+1}}\left(x_{k+1}\right)$, $B_{k+1}$, set $k:=k+1$, and go to step1.

It seems that we have to compute the value of the projected gradient and the objective function twice in every iteration. In practice, $\mu_{k}$ seldom update because it is a sufficient small parameter. Thus, it is rarely to compute the value of the projected gradient and the objective function twice in every iteration. There is an advantage to choosing a large $\Delta_{k+1}=\gamma_{3} \Delta_{k}$ when $\rho_{k} \geq \eta_{2}$, but it may be unwise to 
choose it to be to large, so we give a upper bound $\Delta_{\max }$, and set $\Delta_{k+1}=\min \left\{\Delta_{\max }, \gamma_{3} \Delta_{k}\right\}$ when $\rho_{k} \geq \eta_{2}$.

Observes that the subproblem (6) is compatible during in algorithm, so we do not need any extra feasibility restoration phase[2] in our algorithm, which differentiated our paper from those filter algorithm for nonlinear complementarity problems[2]. By the way, we are surprised to find that $B_{k+1}=\nabla_{x} \Phi_{\mu_{k}}\left(x_{k}\right) \nabla_{x} \Phi_{\mu_{k}}\left(x_{k}\right)^{T}$ can be computed easily instead of updating $B_{k+1}$ with higher numerical expenditure, because of (3) and (7).

\section{CONCLUSIONS AND NUMERICAL EXPERIMENTS}

The new algorithm 1 presented in this paper combines with the multidimensional filter technique and the trust region method, which has a good numerical calculation results.

Now, we give some numerical results for the following 14 complementarity test problems in TABLE I. The values for the constants used in our tests are $\mu_{0}=10^{-5}, \varepsilon=10^{-5}, \gamma_{g}=10^{-3}$, $\gamma_{1}=0.25, \gamma_{3}=2, \eta_{1}=0.25, \eta_{2}=0.95, \theta=0.1, \Delta_{\max }=10^{3}$, $\mathscr{F}_{0}=\left(10^{5}, \ldots, 10^{5}\right)^{\mathrm{T}}$. The trust-region radius update is implemented as

$$
\Delta_{k+1}= \begin{cases}\gamma_{1} \Delta_{k}, & \text { if } \rho_{k}<\eta_{1}, \\ \Delta_{k}, & \text { if } \rho_{k} \in\left[\eta_{1}, \eta_{2}\right), \\ \min \left\{\Delta_{\max }, \gamma_{3} \Delta_{k}\right\}, & \text { if } \rho_{k} \geq \eta_{2} .\end{cases}
$$

Example 1.1 (Kojima-Shindo Nonlinear complementarity test problem)We choose the degenerate example in [2, 3].

Example 1.2 (Kojima-Shindo Nonlinear complementarity test problem)We choose the non-degenerate example in [3].

Example 2 (Kanzow Nonlinear complementarity test problem[2, 4]).

Example 3.1 We choose Example 2 with the constant $b=\left((-1)^{1}, \ldots,(-1)^{i}, \ldots,(-1)^{n}\right)^{T}$ in [5].

Example 3.2 We choose Example 2 with the constant $b=\left((-1)^{1} \sqrt{1}, \ldots,(-1)^{i} \sqrt{i}, \ldots,(-1)^{n} \sqrt{n}\right)^{T}$ in [5].

Example 4 We choose Example 2 in [6].

The computational results are listed in Table 1 , in which iter denotes the number of iterations, and Resf stand for the computing accuracy, i.e. Res $f=f_{\mu_{k}}\left(x_{k}\right)$. The numerical results show that Algorithm 1 is robust and efficient. The number of iterations and computing accuracy for most problems are satisfactory.

TABLE I. NUMERICAL RESULT OF TEST PROBLEMS

\begin{tabular}{|l|l|c|c|}
\hline Example & \multicolumn{1}{|c|}{ Start point } & iter & Re $\boldsymbol{f}$ \\
\hline 1.1 & $(0,0,0,0)^{T}$ & 7 & $4.43 e-12$ \\
\hline 1.1 & $(2,1,0.5,2)^{T}$ & 9 & $1.02 e-12$ \\
\hline 1.2 & $(0,0,0,0)^{T}$ & 9 & $7.98 e-15$ \\
\hline 1.2 & $(2,1,0.5,2)^{T}$ & 8 & $2.84 e-10$ \\
\hline 2 & $(3,2,1,2,3)^{T}$ & 2 & $1.43 e-13$ \\
\hline 2 & $(-2, \ldots,-2)^{T}$ & 3 & $9.17 e-13$ \\
\hline 3.1 & $(2, \ldots, 2)^{T}$ & 8 & $1.66 e-11$ \\
\hline 3.2 & $(2, \ldots, 2)^{T}$ & 5 & $1.05 e-14$ \\
\hline 4 & $\left(10^{-2}, 1,0.5,10^{-2}, 10^{-2}\right)^{T}$ & 4 & $1.68 e-11$ \\
\hline 4 & $(12,-12,12,-12,12)^{T}$ & 9 & $5.84 e-11$ \\
\hline
\end{tabular}

\section{References}

[1] N. I. M. Gould, C. Sainvitu, and P. L. Toint, “A filter-trust-region method for unconstrained optimization,” SIAM J. Optim., vol.16, 2005, pp.341-357.

[2] J. Long and S. Y. Zeng, "A new Filter-Levenberg-Marquardt method with disturbance for solving nonlinear complementarity problems," Appl. Math. Comput., vol. 216, 2010, pp. 677-688.

[3] J. D. Ding and H. Y. Yin, “A new homotopy method for nonlinear complementarity problems,” Numer. Math. J. Chinese Univ. (English Ser.), vol. 16, 2007, pp. 155-163.

[4] T. Ni and P. Wang, "A smoothing-type algorithm for solving nonlinear complementarity problems with a non-monotone line search, "Appl. Math. Comput., vol.216, 2010, pp. 2207-2214.

[5] C. C. Huang and S. Wang, "A power penalty approach to a nonlinear complementarity problem, “ Oper. Res. Letters, vol.38, 2010,pp. 72-76.

[6] Y. Xia, H. Leung, and J. Wang, “A projection neural network and its application to constrained optimization problems," IEEE Transactions on Circuits and Systems-I, vol. 49, 2002,pp. 447-458. 